\title{
JUPITER AS A GIANT COSMIC RAY DETECTOR
}

\author{
P. B. Rimmer, C. R. Stark, and Ch. Helling \\ SUPA, School of Physics \& Astronomy, University of St. Andrews, North Haugh, St. Andrews KY16 9SS, UK; pr33@ st-andrews.ac.uk \\ Received 2014 April 3; accepted 2014 April 28; published 2014 May 14
}

\begin{abstract}
We explore the feasibility of using the atmosphere of Jupiter to detect ultra-high-energy cosmic rays (UHECRs). The large surface area of Jupiter allows us to probe cosmic rays of higher energies than previously accessible. Cosmic ray extensive air showers in Jupiter's atmosphere could in principle be detected by the Large Area Telescope (LAT) on the Fermi observatory. In order to be observed, these air showers would need to be oriented toward the Earth, and would need to occur sufficiently high in the atmosphere that the gamma rays can penetrate. We demonstrate that, under these assumptions, Jupiter provides an effective cosmic ray "detector" area of $3.3 \times 10^{7} \mathrm{~km}^{2}$. We predict that Fermi-LAT should be able to detect events of energy $>10^{21} \mathrm{eV}$ with fluence $10^{-7} \mathrm{erg} \mathrm{cm}^{-2}$ at a rate of about one per month. The observed number of air showers may provide an indirect measure of the flux of cosmic rays $\gtrsim 10^{20} \mathrm{eV}$. Extensive air showers also produce a synchrotron signature that may be measurable by Atacama Large Millimeter/submillimeter Array (ALMA). Simultaneous observations of Jupiter with ALMA and Fermi-LAT could be used to provide broad constraints on the energies of the initiating cosmic rays.
\end{abstract}

Key words: cosmic rays - gamma rays: general - planets and satellites: individual (Jupiter) - submillimeter: general

Online-only material: color figure

\section{INTRODUCTION}

When a cosmic ray proton or nucleus of sufficient energy collides with the nucleus of a molecule in Earth's atmosphere, the result is a shower of particles spread horizontally over kilometers. For example, a $10^{19} \mathrm{eV}$ cosmic ray will produce an extensive air shower spread over a few $\mathrm{km}^{2}$ at sea level. These extensive air showers were independently observed and identified by Rossi (1934) and Auger et al. (1939). The relationship between the depth-dependent composition and the geometrical extent of extensive air showers as well as the composition and energy of the cosmic ray responsible for the primary event can in some cases be determined by reconstructing specific events from observation, such as by the Auger Observatory (Abraham et al. 2010a).

The energy of an extensive air shower is defined here as the energy of the particle responsible for the primary event. The most energetic extensive air showers detected have energies of $E_{0} \sim 10^{20} \mathrm{eV}$. The existence of cosmic rays of $\gtrsim 10^{20} \mathrm{eV}$ would violate the Greisen-Zatsepin-Kuzmin (GZK) limit (Greisen 1966; Zatsepin \& Kuz'min 1966), and explaining the origin and survival of particles at these energies would require either new physics or a nearby source of $\gtrsim 10^{20} \mathrm{eV}$ cosmic rays (Bertolami \& Carvalho 2000). Upper limits for the cosmic ray flux at these energies is severely detector limited, and the probability of detecting events $>10^{20} \mathrm{eV}$ becomes vanishingly small.

Are there cosmic rays of energy $\gg 10^{20} \mathrm{eV}$, and if so, how can they be detected? Possible indirect detection strategies for cosmic rays over a wide range of energies involve gamma ray observations of, e.g., the Galactic center (Su et al. 2010), regions of dark matter (Sciama 2000), and molecular clouds (Casanova et al. 2010).

Atmospheres of planets and stars could themselves act as large detectors for indirect detection of very energetic cosmic ray events. The cosmic ray induced gamma ray emission in Earth's atmosphere has been mapped by Petry (2005) and has recently been measured by Fermi in order to constrain the cosmic ray spectrum between $90 \mathrm{GeV}$ and $6 \mathrm{TeV}$ (Ackermann et al. 2014). Searching for energetic cosmic ray events in the atmosphere of our Sun is difficult because the Sun itself is the source of energetic particles as well as gamma radiation (e.g., Kanbach et al. 1993). Nevertheless, quiescent solar gamma radiation is detected by the Fermi satellite, and is attributed to cosmic rays (Orlando et al. 2009; using the model of Seckel et al. 1991).

Jupiter is a natural candidate for remote detection of extensive air showers because it is a large dense object that is not expected to produce its own gamma ray emission. Jupiter has been seen in auroral emission, due to a complex interplay between volcanic particulates from Io, the Jovian magnetosphere and the solar wind (see, e.g., Kim et al. 1998). There is a tentative gamma ray detection from Jupiter, setting a flux limit of $6.3 \times 10^{-7} \mathrm{erg} \mathrm{cm}^{-2} \mathrm{~s}^{-1}$ (Fichtel et al. 1975). The cause of this emission is unknown. de Pater (2004) has measured the radio synchrotron variability on Jupiter with LOFAR, and this variability is similar to the variability observed in brown dwarfs (Heinze et al. 2013).

In this Letter, we explore the feasibility of treating Jupiter as a detector of galactic cosmic ray events of energy $E_{0}>$ $10^{20} \mathrm{eV}$. In Section 2 we estimate how often these cosmic ray events can occur within Jupiter's atmosphere. We discuss the electromagnetic component of the shower in a model atmosphere of Jupiter in Section 3. In Section 4, we discuss gamma ray emission (Section 4.1) and synchrotron emission (Section 4.2) from events occurring within Jupiter's atmosphere. Section 5 contains our concluding remarks on the feasibility of detecting these signatures.

\section{OCCURRENCE OF EXTENSIVE AIR SHOWERS}

In order to explore the frequency of extensive air showers on Jupiter, we first need to determine the integrated flux of cosmic rays of primary energy $E_{0}>10^{18} \mathrm{eV}$, called ultra-high-energy cosmic rays (UHECRs). The flux-density for primary cosmic rays of $E_{0}<4 \times 10^{19} \mathrm{eV}$ can be fitted by $j\left(E_{0}\right) \propto E_{0}^{-2.7}$ 
(Beringer et al. 2012; their Equation (26.2)). There is evidence that, above this energy, the assumed power law becomes steeper (Abraham et al. 2008). We therefore use a broken power-law spectrum kinked at $E_{10}=4 \times 10^{10} \mathrm{GeV}$ :

$$
j_{p}\left(E_{0}\right) \approx j_{0} \begin{cases}E_{0}^{-\epsilon}, & E_{0}<E_{10} \\ E_{10}^{\kappa-\epsilon} E_{0}^{-\kappa}, & E_{0}>E_{10}\end{cases}
$$

where $j_{0}=1.8 \mathrm{~cm}^{-2} \mathrm{~s}^{-1} \mathrm{sr}^{-1} \mathrm{GeV}^{-1}, \epsilon=2.7, \kappa=4.2$, and $\left[E_{0}\right]=\mathrm{GeV}$.

We can use this flux density to determine a frequency of extensive air showers above a given energy. Since we are interested in the highest energy cosmic rays, we calculate the frequency for extensive air shower events over a "detector" with surface area $A \Omega\left(\mathrm{cm}^{2} \mathrm{sr}\right), v_{\text {EAS }}(\mathrm{s})$, expressed as

$$
\nu_{\mathrm{EAS}}=A \Omega \int_{E_{0}}^{\infty} j_{p}(E) d E .
$$

For the Pierre Auger Observatory, with a surface area of roughly $A \Omega \approx 2 \pi 3000 \mathrm{~km}^{2}$, the average amount of time between two $4 \times 10^{19} \mathrm{eV}$ events is about 1.5 days. For Jupiter, $A \Omega \approx 4 \pi R_{J}^{2}$, where $R_{J}=6.99 \times 10^{9} \mathrm{~cm}$ is Jupiter's radius, and the time between $4 \times 10^{19} \mathrm{eV}$ events is about $0.1 \mathrm{~s}$. We will not be able to observe the direct consequences of all extensive air showers occurring in Jupiter's atmosphere. The effective detector area is calculated for gamma ray observations in Section 4.1. This effective area will depend on integrated column densities through Jupiter's atmosphere, and therefore on the temperature profile of Jupiter.

\section{ATMOSPHERIC AND EXTENSIVE AIR SHOWER MODEL FOR JUPITER}

We now apply Heitler's approximation for an extensive air shower (Matthews 2005) to a model of Jupiter's atmosphere. We use the one-dimensional temperature profile for the $30^{\circ} \mathrm{N}$ latitude vernal equinox model for Jupiter from Moses et al. (2005), and we make the assumption that Jupiter's temperature profile is isotropic. The profile from Moses et al. (2005) was constructed mostly from observations, at pressures $>10^{-3}$ bar from infrared observations from the Infrared Space Observatory and at pressures $<10^{-6}$ bar from the Galileo probe. Between $10^{-6}$ bar and $10^{-3}$ bar, Moses et al. (2005) assume isothermal temperatures. This temperature profile and bulk chemistry have an effect on the development of extensive air showers.

The penetration depth for an extensive air shower is parameterized by the shower age in terms of the column density $X$, $s=s(X)$, such that $s=0$ corresponds to the initiating event, $s=1$ corresponds to the shower maximum, and $s \rightarrow 3$ as $X \rightarrow \infty$. The relationship between $s$ and $X$ depends on various atmospheric properties. The shower age can be fit with a broken power law (Abraham et al. 2010b):

$$
s(X)= \begin{cases}\frac{3}{1+\frac{2 \chi_{0}}{X} \log \left(\frac{E_{0}}{E_{\text {crit }}}\right)}, & E_{0} \leqslant E_{9} ; \\ \frac{3}{1+\frac{2 \chi_{0}^{\prime}}{X}\left|\log \left(\frac{E_{0}}{E_{\text {crit }}}\right)\right|^{0.35},} & E_{0}>E_{9} ;\end{cases}
$$

where $E_{9}=2 \times 10^{18} \mathrm{eV}, \chi_{0}$ is the radiation path length and $\chi_{0}^{\prime}$ is given its value such that $s(X)$ is continuous over $E_{9}$. The values of the parameters $r_{M}, E_{\text {crit }}$, and $\chi_{0}$ are taken from the Particle Data Group, pdg.lbl.gov, with the atmospheric chemical and physical properties taken from Moses et al. (2005). For this model atmosphere, $E_{\text {crit }}=3.448 \times 10^{8} \mathrm{eV}$ is the critical energy, and $\chi_{0}=63.04 \mathrm{~g} \mathrm{~cm}^{-2}$ and $\chi_{0}^{\prime}=277.2 \mathrm{~g} \mathrm{~cm}^{-2}$ are characteristic path lengths.

The number of electrons in an extensive air shower, $N_{e}$, has been related to the energy of the initiating cosmic ray by Nagano et al. (1992, their Equation (2.3)). We use Heitler's approximation for the number of electrons at the shower maximum (Matthews 2005):

$$
N_{e} \approx \frac{E_{0}}{10 E_{\text {crit }}} \text {. }
$$

The normalized energy distribution of secondary electrons is independent of the energy and composition of the initial cosmic ray, according to CORSIKA simulations (Nerling et al. 2006). The analytic function for the distribution is (Nerling et al. 2006; their Equation (9))

$$
\eta(s, E)=\frac{k_{0} E e^{k_{1} s-k_{2} s^{2}}}{(E+6.43-1.53 s)(E+168 .-42.1 s)^{s}},
$$

where the parameters $k_{0}=0.142, k_{1}=6.180$, and $k_{2}=0.606$, and $s(X)$ is the shower age. We use these calculations for hypothetical air showers in Jupiter's atmosphere in order to estimate their gamma ray and synchrotron signatures.

\section{DIRECT OBSERVATION OF EXTENSIVE AIR SHOWERS ON JUPITER}

We explore the feasibility of observing extensive air showers in Jupiter's atmosphere by gamma ray emission (Section 4.1) and Synchrotron emission (Section 4.2).

\subsection{Gamma Ray Emission}

Extensive air showers begin with $\pi^{+}, \pi^{-}$, and $\pi^{0}$ particles. The $\pi^{0}$ particle decays quickly into $2 \gamma$. After traveling a characteristic column on the order of the radiation length, the gamma rays will catastrophically produce $e^{+} e^{-}$pairs. These electrons in turn produce photons via Bremsstrahlung emission (Gaisser 1990). We adopt the approximation of Matthews (2005) and estimate that the number of photons from an electromagnetic sub-shower is $\sim 10 \times$ the number of electrons, keeping in mind that the flux spectrum produced by Bremsstrahlung is fairly flat up to the energy of the electron. We therefore make the crude approximation that photons spanning an energy of $\Delta E_{\gamma}$ are of number $N_{\gamma} \approx N_{e}\left(E_{e}\right)$ for all $E_{\gamma}<E_{e}$. We can determine $N_{e}\left(E_{e}\right)$ from Equations (4) and (5). These photons are spread over an area the size of $\pi\left(\ell \tan \theta_{c}\right)^{2}$, where $\ell(\mathrm{cm})$ is the distance through the atmosphere between the initiating event for the air shower and the observer, which we take to be $\sim 5 \mathrm{AU}$, and $\theta_{c}$ is the opening angle of the shower (Figure 1). The statistical rms of the angle between the axis of the shower and the photons is $\theta_{c} \approx m_{e} c^{2} / E_{e}$ (Gaisser 1990). The gamma ray fluence, $\Phi$, for an extensive air shower is approximately

$$
\Phi \approx \int_{\Delta E_{\gamma}} \frac{N_{\gamma}\left(E_{\gamma}\right) d E_{\gamma}}{\pi \ell^{2} \tan ^{2}\left(m_{e} c^{2} / E_{e}\right)},
$$

where the integral is taken over the range of energies relevant for a given gamma ray detector, $\Delta E_{\gamma}(\mathrm{eV})$. Because the fluence depends on the square of $\tan \theta_{c}$, which for small angles is $\sim\left(E_{e} / m_{e} c^{2}\right)^{2}$, the highest energy electrons in the distribution (Equation (5)) will determine the maximum fluence. 


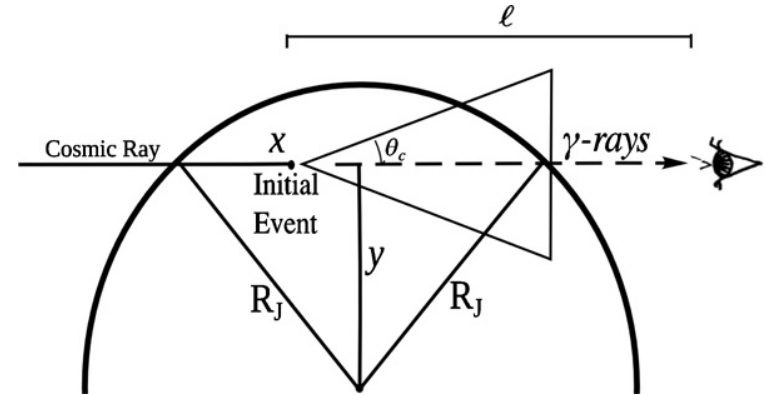

Figure 1. Diagram of a cosmic ray initiating an extensive air shower and producing photons on a trajectory toward an observer on Earth. Cartesian coordinates are oriented such that the $x$-axis is along the path of the cosmic ray and extensive air shower. $R_{J}=6.9911 \times 10^{9} \mathrm{~cm}$ denotes the radius of Jupiter.

The maximum fluence is only observed if the center of the gamma ray shower strikes the detector. The gamma rays produced by the highest energy electrons are effectively pencil beams, and so it is more likely to observe the gamma ray component at less than the maximum fluence. We will now consider the effective "detector area" for gamma ray emission from extensive air showers in Jupiter's atmosphere, and then we will return to the question of whether Fermi Large Area Telescope (LAT) can detect these showers.

Some extensive air showers will be directed toward the Earth, and will not have traveled through so much of Jupiter's atmosphere as to be significantly attenuated. If Jupiter is thought of as a giant cosmic ray detector, we can use this fact to estimate the effective detector area that Jupiter provides for observing cosmic rays. In order to make this estimate, we need to consider cosmic rays transiting through a ring in the upper atmosphere of Jupiter bookended by two column densities. For column densities below $\sim 30 \mathrm{~g} \mathrm{~cm}^{-2}$, it is unlikely that UHECRs will experience a collision, and so the cosmic rays will stream through the atmosphere unaffected. For column densities much above $\sim 710 \mathrm{~g} \mathrm{~cm}^{-2}$, the gamma ray signature from an extensive air shower will be attenuated by the atmosphere (Alvarez-Muniz et al. 2002; Beringer et al. 2012). Extensive air showers will therefore be remotely observable at a range of column densities $30 \mathrm{~g} \mathrm{~cm}^{-2} \lesssim X_{\perp} \lesssim 710 \mathrm{~g} \mathrm{~cm}^{-2}$. Above $710 \mathrm{~g} \mathrm{~cm}^{-2}$, we assume complete attenuation, and below $710 \mathrm{~g} \mathrm{~cm}^{-2}$ we assume no attenuation (i.e., we assume the best-case scenario, that the shower maximum is achieved just before the shower exits the atmosphere of Jupiter). We calculate $X_{\perp}$ using the density profile for Jupiter from Moses et al. (2005) as follows.

We can solve for the column density experienced by a cosmic ray penetrating into Jupiter, with its closest approach to the center of Jupiter a distance $y(\mathrm{~cm})$. A diagram of the cosmic ray path is shown in Figure 1. The column density is obtained by solving the integral

$$
X_{\perp}(y)=\int_{-\sqrt{R_{J}^{2}-y^{2}}}^{\sqrt{R_{J}^{2}-y^{2}}} d x n(h(x, y)),
$$

where $n(h)\left(\mathrm{cm}^{-3}\right)$ is the number density in Jupiter's atmosphere at a height $h(x, y)=\sqrt{x^{2}+y^{2}}$. The solution of this integral for various values of $y$ is plotted in Figure 2. The effective "detector" for the Jovian atmosphere is a ring with a surface area of $A \Omega \approx 2 r R_{J} \Omega$, where $r \approx 10^{-3} R_{J}$. The solid angle, $\Omega$, through which the cosmic ray must travel in order to produce an air shower that Fermi can detect is restricted by the opening angle, $\theta_{c}$. The angular spread of extensive air showers

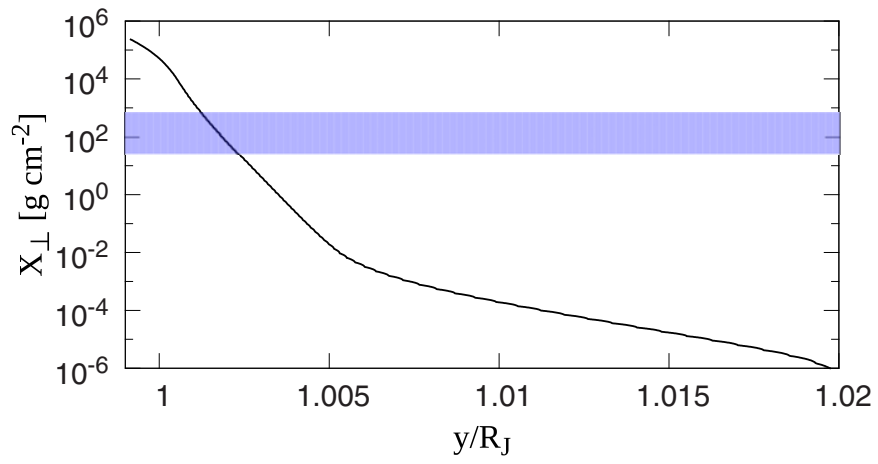

Figure 2. Integrated column density, $X_{\perp}\left(\mathrm{g} \mathrm{cm}^{-2}\right)$ vs. the ratio $y / R_{J}$. The blue shaded region represents the range of column densities where gamma rays from cosmic ray air showers could in principle be detected.

(A color version of this figure is available in the online journal.)

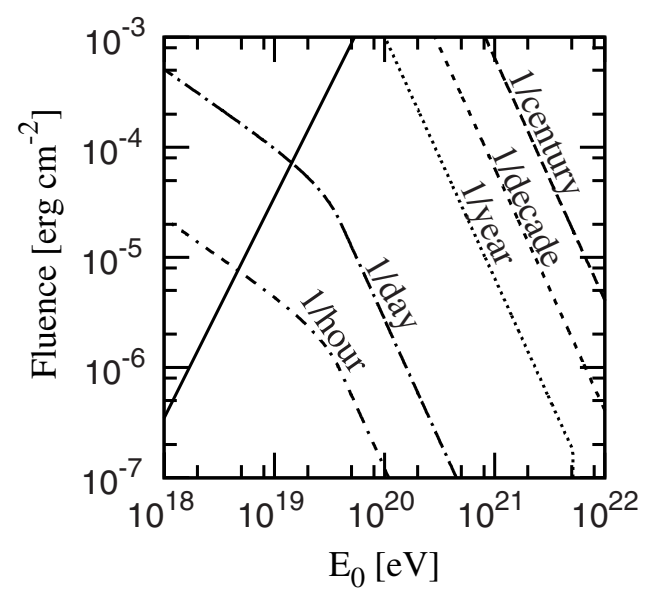

Figure 3. Contour plot of cosmic ray frequency as a function of the energy of the extensive air-shower, $E_{0}(\mathrm{eV})$, and the gamma ray fluence $\left(\mathrm{erg} \mathrm{cm}^{-2}\right)$ as observed by Fermi-LAT. The solid line indicates the maximum fluence from an extensive air shower of energy $E_{0}$.

occurring in the region $30 \mathrm{~g} \mathrm{~cm}^{-2} \lesssim X_{\perp} \lesssim 710 \mathrm{~g} \mathrm{~cm}^{-2}$ projects a ring at a distance $\ell$ from Jupiter with surface area $A \Omega \approx 4 \pi \ell R_{J} m_{e} c^{2} / E_{e}$.

We now compare the gamma ray fluence from an event in Jupiter's atmosphere, $\Phi$, to the detection limit for gamma ray fluence for Fermi's Gamma-ray Burst Monitor (GBM) and Fermi-LAT using the sensitivity estimates and the spectral range from Meegan et al. (2009) and Atwood et al. (2009), respectively. Fermi-GBM observes gamma rays in an energy range of $\Delta E_{\gamma}=0.008-10 \mathrm{MeV}$ and a sensitivity of $\sim 10^{-5} \mathrm{erg} \mathrm{cm}^{-2}$, and Fermi-LAT with $\Delta E_{\gamma}=0.02-300 \mathrm{GeV}$ and a sensitivity of $\sim 10^{-7} \mathrm{erg} \mathrm{cm}^{-2}$. At its highest fluence sensitivity, Fermi-LAT can achieve an angular resolution of $\sim 0.5$.

The detection limits for gamma ray fluence can be applied to Equation (6) in order to solve for $E_{e}$. The effective cosmic ray detector area for Jupiter, as observed by Fermi, is

$$
A \Omega=\frac{2}{5}\left(\frac{R_{J} \Delta E_{\gamma}}{\ell \Phi}\right)\left(\frac{E_{0}}{m_{e} c^{2}}\right) \leqslant 4 \pi r R_{J} .
$$

We now solve Equation (2), using Equation (8) for $A \Omega$. Our resulting cosmic ray detection frequency for Fermi-LAT is presented as a function of the gamma ray fluence, $\Phi$, and the energy of the extensive air shower, in Figure 3. We predict that Fermi-LAT should be able to detect events of energy $>10^{21} \mathrm{eV}$ with fluence $10^{-7} \mathrm{erg} \mathrm{cm}^{-2}$ at a rate of about one per month. Due 


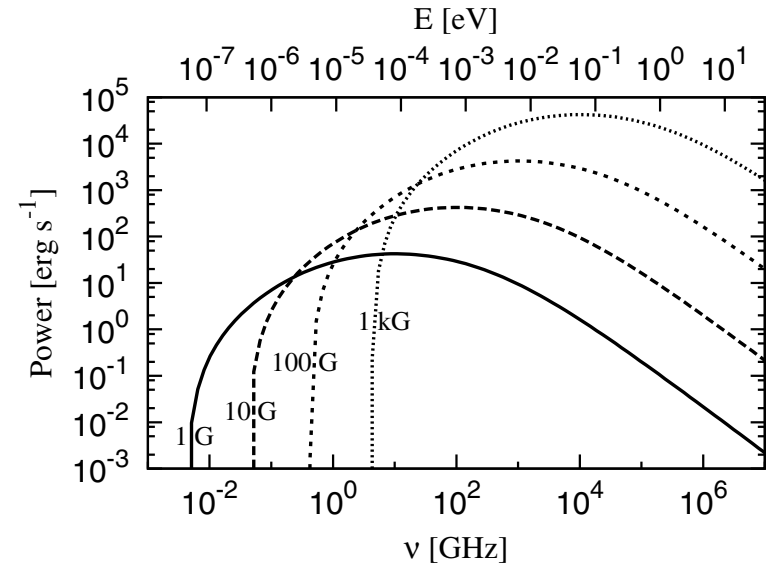

Figure 4. Synchrotron emission power ( $\mathrm{erg} \mathrm{s}^{-1}$ ) for extensive air showers of energy $10^{18} \mathrm{eV}$, at four different magnetic field strengths, ranging from $1 \mathrm{G}$ to $1 \mathrm{kG}$, as a function of frequency $v\left(\mathrm{~s}^{-1}\right)$.

to its lower energy range, we do not predict that Fermi-GBM will be able to detect any extensive air showers from Jupiter.

\subsection{Synchrotron Emission}

The electronic component of an extensive air shower would have a characteristic spectroscopic signature due to synchrotron emission. We can calculate an approximate power-spectrum for the synchrotron emission from the relativistic electrons within our extensive air shower.

Imagine a number of electrons, $\mathcal{N}_{\gamma}$, each in the same magnetic field, $B$, and each with the same velocity characterized by $\gamma=\left(1-v^{2} / c^{2}\right)^{-1 / 2}$. These electrons will all radiate at an identical synchrotron frequency (Felten \& Morrison 1966, their Equation (29)):

$$
v\left(\mathrm{~s}^{-1}\right) \approx \frac{3 e \gamma^{2} B}{4 \pi m_{e} c},
$$

and will radiate with a power (Felten \& Morrison 1966, their Equation (32)):

$$
P\left(\operatorname{erg~s}^{-1}\right)=\frac{2 \gamma^{2} e^{4} B^{2} \mathcal{N}_{\gamma}^{2}}{3 m_{e}^{2} c^{3}} .
$$

Given a distribution of electrons, $\eta(E)$, from Equation (5), we can determine the power as a function of the frequency. The relativistic factor can be given as a function of the energy, $\gamma=E /\left(m_{e} c^{2}\right)+1$, and we can give the electron energy in terms of the synchrotron frequency produced by electrons of that energy:

$$
E(\nu)=m_{e} c^{2}\left(\sqrt{\frac{4 \pi m_{e} c \nu}{3 e B}}-1\right) .
$$

The number of free electrons that will produce a synchrotron signature at the frequency $v$ is then given by $\mathcal{N}_{\gamma}=N_{e} \eta(\nu)$. For an extensive air shower, $N_{e}$ from Equation (4) and $\eta(\nu)$ comes from Equation (5) with the energy from Equation (11). The radiation power is then

$$
P(v)=\frac{8 \pi e^{3} B N_{e}^{2}}{9 m_{e} c^{2}} v \eta^{2}(E(v)) .
$$

The resulting synchrotron spectrum for extensive air showers is plotted as a function of $v$ in Figure 4. Our results compare

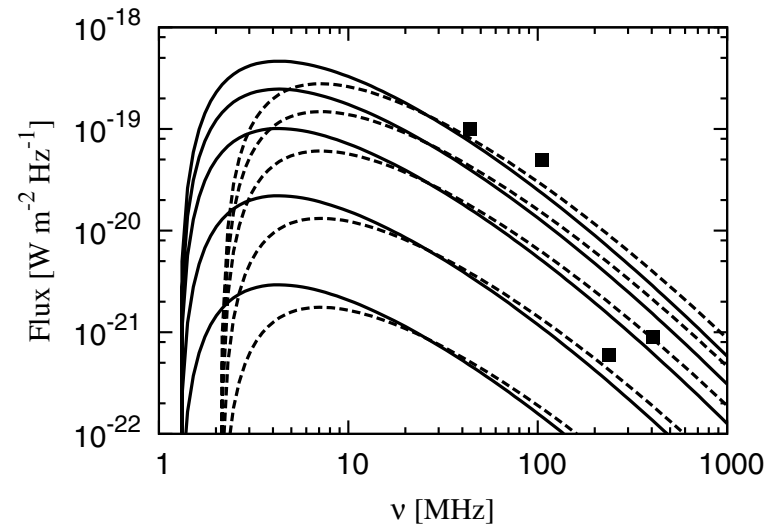

Figure 5. Synchrotron emission flux $\left(\mathrm{W} \mathrm{m}^{-2} \mathrm{~Hz}^{-1}\right)$ for extensive air showers of energy $10^{16}-10^{17} \mathrm{eV}$, as a function of frequency, $v\left(\mathrm{~s}^{-1}\right)$ for magnetic field strengths of $0.3 \mathrm{G}$ (solid lines) and $0.5 \mathrm{G}$ (dashed lines). These spectra are compared to the data from Spencer (1969, black points).

well with both the synthetic spectrum and radio observations presented in Falcke \& Gorham (2003), if the ambient magnetic field is set to 0.3 G. Falcke \& Gorham (2003) fit their synthetic spectrum (their Figure 2) to the data from Spencer (1969). The data can be well fit by cosmic rays of energies $10^{16} \mathrm{eV}-10^{17} \mathrm{eV}$. Our comparison with the data from Spencer (1969) for magnetic field strengths of $0.3-0.5 \mathrm{G}$ is plotted in Figure 5. This comparison demonstrates the variation of the synchrotron power spectrum with cosmic ray energy.

Jupiter's magnetic field of $B_{J} \sim 4 \mathrm{G}$ will shift the peak frequency of the synchrotron emission to approximately $6 \mathrm{~mm}$, bringing the synchrotron signature into the detection range of the Atacama Large Millimeter/submillimeter Array. Although it may be virtually impossible to disentangle the synchrotron emission from extensive air showers from other microwave emission on Jupiter, coincident detections could be achieved by simultaneously observing Jupiter with a millimeter telescope and the Fermi-LAT.

Since the peak frequency of the synchrotron emission depends on the energy distribution of the electrons, and this distribution is expected to be independent of the cosmic ray energy (Nerling et al. 2006), the peak frequency of this emission will depend on the local magnetic field. If the local magnetic field where the extensive air showers occurs is different from $B_{J}$, then the peak frequency will shift as shown in Figure 4. Radio and infrared synchrotron emission from events on Jupiter is therefore also possible.

\section{CONCLUDING REMARKS}

We suggest observing Jupiter for signatures of very high energy cosmic ray events, first by considering how often extensive air showers occur on the surface of Jupiter, and then by examining the gamma ray and synchrotron signatures of such an air shower. If gamma ray observations could be connected to extensive air showers in Jupiter's atmosphere, this would afford the cosmic ray community a significantly larger detector area and could help constrain the cosmic ray spectrum above $10^{20} \mathrm{eV}$. We can apply Jupiter's "detector" cross-section of $3 \times 10^{7} \mathrm{~km}^{2}$ to Equation (2) to estimate an expected cosmic ray event frequency. If the observed number of events significantly exceeded one every $12 \mathrm{hr}$, this would be evidence of GZK violation, where cosmic rays survive at energies greater than those at which interaction with the blueshifted cosmic microwave background 
becomes significant. This would imply either a local source of $>10^{20} \mathrm{eV}$ cosmic rays or new physics. We suggest using the Fermi observatory to observe this gamma ray signature.

Brown dwarfs are comparable to Jupiter in size. Since brown dwarfs are far more distant than Jupiter, gamma ray detection of extensive air showers in their atmospheres would be unlikely in the foreseeable future. Nevertheless, it may be possible to observe the synchrotron emission from the electrons comprising extensive air showers, and the occurrence of extensive air showers in atmospheres of free-floating substellar objects, far from any host star, may be an important source of electrification within their cloud layers. We will examine these possibilities in a later paper.

All authors highlight financial support of the European Community under the FP7 by an ERC starting grant. P.B.R. is grateful for critical corrections from Alan Watson on an earlier draft, Alexander MacKinnon for helpful discussions, and Ian Taylor for his technological support. We thank the anonymous referee for their helpful comments. This research has made use of NASA's Astrophysics Data System.

\section{REFERENCES}

Abraham, J., Abreu, P., Aglietta, M., et al. 2010a, PhLB, 685, 239 Abraham, J., Abreu, P., Aglietta, M., et al. 2008, PhRvL, 101, 061101 Abraham, J., Abreu, P., Aglietta, M., et al. 2010b, PhRvL, 104, 091101
Ackermann, M., Ajello, M., Albert, A., et al. 2014, PhRvL, 112, 151103

Alvarez-Muniz, J., Engel, R., Gaisser, T. K., Ortiz, J. A., \& Stanev, T. 2002, PhRvD, 66, 033011

Atwood, W. B., Abdo, A. A., Ackermann, M., et al. 2009, ApJ, 697, 1071

Auger, P., Ehrenfest, P., Maze, R., Daudin, J., \& Fréon, R. A. 1939, RvMP, 11,288

Beringer, J., Arguin, J.-F., Barnett, R. M., et al. 2012, PhRvD, 86, 010001

Bertolami, O., \& Carvalho, C. S. 2000, PhRvD, 61, 103002

Casanova, S., Aharonian, F. A., Fukui, Y., et al. 2010, PASJ, 62, 769

de Pater, I. 2004, P\&SS, 52, 1449

Falcke, H., \& Gorham, P. 2003, APh, 19, 477

Felten, J. E., \& Morrison, P. 1966, ApJ, 146, 686

Fichtel, C. E., Hartman, R. C., Kniffen, D. A., et al. 1975, ApJ, 198, 163

Greisen, K. 1966, PhRvL, 16, 748

Gaisser, T. K. 1990, Cosmic Rays and Particle Physics (Cambridge Univ. Press)

Heinze, A. N., Metchev, S., Apai, D., et al. 2013, ApJ, 767, 173

Kanbach, G., Bertsch, D. L., Fichtel, C. E., et al. 1993, A\&AS, 97, 349

Kim, S. J., Lee, D. H., \& Kim, Y. H. 1998, RPPh, 61, 525

Matthews, J. 2005, APh, 22, 387

Meegan, C., Lichti, G., Bhat, P. N., et al. 2009, ApJ, 702, 791

Moses, J., Fouchet, T., Bézard, B., et al. 2005, JGRE, 110, E08001

Nagano, M., Teshima, M., Matsubara, Y., et al. 1992, JPhG, 18, 423

Nerling, F., Blümer, J., Engel, R., \& Risse, M. 2006, APh, 24, 421

Orlando, E., \& for the Fermi Large Area Telescope Collaboration. 2009, Proc. 31st ICRC (Lodz), arXiv:0907.0557

Petry, D. 2005, in AIP Conf. Proc. 745, High Energy Gamma-Ray Astronomy, ed. F. A. Aharonian, H. J. Volk, \& D. Horns (Melville, NY: AIP), 709

Rossi, B. 1934, Supplemento a la Ricerca Scientifica, 1, 579

Sciama, D. W. 2000, MNRAS, 312, 33

Seckel, D., Stanev, T., \& Gaisser, T. K. 1991, ApJ, 382, 652

Spencer, R. E. 1969, Natur, 222, 460

Su, M., Slatyer, T. R., \& Finkbeiner, D. P. 2010, ApJ, 724, 1044

Zatsepin, G. T., \& Kuz'min, V. A. 1966, JETPL, 4, 78 